\title{
Simulation numérique d'un jet turbulent axisymétrique à masse volumique variable par le modele au second ordre (RSM)
}

\author{
Mohammed Senouci ${ }^{\mathrm{a}}$ et Abdelhamid Bounif \\ Faculté de Génie Mécanique-Université des Sciences et de la Technologie d’Oran Mohamed Boudiaf, Laboratoire LCGE, \\ BP 1505, 31000 Elmnaouar, Algérie
}

Reçu le 13 avril 2011, accepté le 11 mai 2011

\begin{abstract}
Résumé - Dans le présent article, les jets turbulents confinés axisymétriques non réactifs sont étudiés en utilisant le modèle de turbulence de second ordre. L'objectif est de mettre en évidence, dans la région initiale du jet, les effets de la variation de densité, provoquée par un écart de température entre le jet d'air chauffé et l'air ambiant, sur les principaux paramètres caractéristiques de tels écoulements. Les résultats numériques obtenus sont alors discutés en comparaison avec ceux issus des expériences de Djeridane [T. Djeridane, Contribution à l'étude expérimentale de jets turbulents axisymétriques à densité variable, Thèse de doctorat, Univ. Aix-Marseille II, 1994] (relatifs aux jets air/air et He/air) et d'autres trouvées dans la littérature. Les mesures expérimentales de la vitesse longitudinale, de l'écart-type des tensions de Reynolds et du scalaire, présentent qualitativement le même comportement. L'écart entre la simulation numérique et l'expérience n'est pas important et les résultats numériques obtenus sont en général satisfaisants.
\end{abstract}

Mots clés : Turbulence / jets / effets de densité / modélisation au second ordre

\begin{abstract}
Numerical simulation of an axisymmetric turbulent jet with variable density using a Reynolds stress model. In this paper, the nonreacting axisymmetric confined turbulent jets are studied by using the second order turbulence model. The aim is to present, in the initial region of the jet, the variable density effects, caused by a difference in temperature between the hot air and the ambient air, on the principal characteristic parameters of such flows. The numerical results obtained are then discussed in comparison with experimental data Djeridane [T. Djeridane, Contribution à l'étude expérimentale de jets turbulents axisymétriques à densité variable, Thèse de doctorat, univ. Aix-Marseille II, 1994] (relating to the air/air and He/air jets) and other found in the literature. Experimental measurements of the axial velocity, the Reynolds stress and the scalar, present qualitatively the same behaviour. The comparison of the numerical simulation to the experiment does not show large difference and the results obtained are in general satisfactory.
\end{abstract}

Key words: Turbulence / axisymmetric jets / density effects / second order modelling

\section{Introduction}

La majorité des écoulements, d'intérêt technologique, sont turbulents et à masse volumique variable : chambre de combustion, production d'énergie, le jet qui s'échappe d'un turboréacteur, rejets de gaz dans l'atmosphère, etc. La variation de la masse volumique peut être provoquée soit par écart de température au sein d'un seul fluide soit par un mélange, sans réaction chimique, de deux fluides miscibles de densités différentes. Fondamentalement, la complexité de ces écoulements tient au fait que les

\footnotetext{
a Auteur pour correspondance : med_snci@yahoo.fr
}

équations du champ dynamique sont fortement couplées à celles du champ scalaire via une équation d'état reliant la quantité scalaire responsable des variations de densité à celle-ci. L'étude des écoulements turbulents à masse volumique variable présente donc, tant sur un plan fondamental que sur un plan industriel, un grand intérêt.

L'étude numérique, qui a pour objet de tester et de développer différentes modélisations pour s'approcher le plus précisément possible de l'expérience tout en préservant l'universalité des modèles développés, a pour base les modèles de turbulence avec fermeture sur les moments du second ordre. Ce niveau de fermeture apparaît 


\section{Nomenclature}

\begin{tabular}{|c|c|}
\hline \multicolumn{2}{|r|}{ Lettres romaines : } \\
\hline$\overline{a_{i j}}$ & Tenseur d'anisotropie \\
\hline$D_{i j}$ & Diffusion turbulente des tensions de Reynolds \\
\hline$D_{\varepsilon}$ & Diffusion turbulente de la dissipation de l'énergie cinétique de la turbulence \\
\hline$D_{j}$ & Diamètre de la buse \\
\hline$F$ & Scalaire instantané \\
\hline$k$ & Énergie cinétique de la turbulence \\
\hline$M$ & Flux de quantité de mouvement \\
\hline$p$ & Pression instantanée \\
\hline$P_{i j}$ & Production de $R_{i j}$ (tensions de Reynolds) par action du champ dynamique moyen \\
\hline $\operatorname{Pr}$ & Nombre de Prandt \\
\hline$R e_{\mathrm{j}}$ & Nombre de Reynolds du jet $R e_{\mathrm{j}}=\frac{U_{j} D_{j}}{v_{j}}$ \\
\hline$r$ & Distance à l'axe du jet \\
\hline$R_{d}$ & Rapport des densités entre le jet et le fluide ambiant $R_{d}=\rho_{\mathrm{j}} / \rho_{\mathrm{a}}$ \\
\hline$T$ & Température instantanée \\
\hline$u_{i}, u_{i}^{\prime \prime}$ & Composante $i$ des fluctuations au sens de Favre et de Reynolds de la grandeur $U_{i}$ \\
\hline$U, V$ & Vitesses longitudinale et radiale instantanées \\
\hline$x$ & Distance à la buse \\
\hline \multicolumn{2}{|r|}{ Lettres grecques: } \\
\hline$\varepsilon$ & Dissipation de l'énergie cinétique de la turbulence \\
\hline$\Phi$ & Variable \\
\hline$\nu$ & Viscosité cinématique \\
\hline$\rho$ & Masse volumique \\
\hline$\delta_{i j}$ & Symbole de Kronecker \\
\hline \multicolumn{2}{|r|}{ Conventions } \\
\hline- & Moyenne au sens de Reynolds (non pondérée) \\
\hline$\sim$ & Moyenne au sens de Favre (pondérée par la masse) \\
\hline$(.)^{\prime}$ & Écart-type \\
\hline$(.)_{j}$ & Référence à la sortie de la buse $(x=0)$ \\
\hline$(.)_{i j}$ & Notation tensorielle avec sommation sur les indices répétés \\
\hline
\end{tabular}

comme étant le niveau minimum où une approche physique réaliste des termes présents dans les équations peut être développée, les modèles d'ordres inférieurs constituant alors des simplifications des modèles du second ordre dans l'optique d'utilisations plus restrictives.

Malgré les difficultés encore rencontrées pour les écoulements à masse volumique constante, il apparaît nécessaire de bien comprendre les effets induits par les variations de densité sur les écoulements turbulents et de développer des modélisations capables de les prédéterminer. Jusqu'à présent, la voie la plus largement utilisée pour la prédétermination numérique des écoulements turbulents à masse volumique variable a été celle indiquée par Favre [2]. Celle-ci est basée sur l'utilisation d'une moyenne pondérée par la masse pour décomposer toutes les variables instantanées de l'écoulement, autre que la densité et la pression, en une partie macroscopique moyenne et une partie fluctuante. D'un point de vue physique, l'utilisation de cette moyenne est principalement justifiée par le fait qu'une grandeur essentielle pour le mouvement d'un fluide lorsque la masse volumique varie n'est plus uniquement la vitesse, comme dans le cas à densité constante, mais la quantité de mouvement. Des simulations au second ordre en moyenne de
Favre ont été réalisées pour ce type d'écoulement par Harran [3], Joly [4] et Sanders [5]. Dans le même ordre de travaux, il faut citer aussi l'étude réalisée par Ruffin [6] en liaison très proche avec l'expérimentation menée par Djeridane [1] pour un jet confiné. Cette étude permet de montrer clairement que le paramètre principal qui gouverne les échelles caractéristiques de la turbulence est le flux de la quantité de mouvement imposé à la section d'éjection de ces jets. On peut aussi citer l'étude expérimentale réalisée par Pagé [7] et plus récemment les études réalisées par Chassaing [8] et Gupta [9]. Pour le cas d'un jet libre, on peut citer par exemple, les études numériques réalisées par Sanders [10], Harran [11] et Joly [12] ainsi que les mesures complémentaires de Djeridane [1] effectuées dans les jets d'air et d'hélium et plus récemment, les études réalisées par Mi [13] et Gupta [9]. Dans son étude, consacrée particulièrement à l'étude de l'évolution des champs scalaires en utilisant l'équation de transport pour le taux de dissipation scalaire, au lieu d'une expression algébrique, Sanders [10] a montré que l'utilisation d'un modèle algébrique est suffisant pour le calcul des jets libres et qu'une équation de transport pour le taux de dissipation scalaire est certainement préférable par rapport à un modèle algébrique pour 
calculer les écoulements plus complexes qu'un jet turbulent libre. Djeridane [1], dans ses mesures, a trouvé que ce type de jets est caractérisé par un épanouissement plus rapide du champ dynamique et un niveau de la turbulence légèrement faible comparé aux jets turbulents confinés.

Dans la présente étude, les effets de la variation de densité sur les principaux paramètres caractéristiques d'un écoulement de jet turbulent confiné axisymétrique, sont étudiés en utilisant le modèle de turbulence de second ordre en moyenne de Favre. La quantité scalaire responsable des variations de densité est la température.

\section{2 Équations de conservation}

Le système d'équations obtenu, pour un écoulement stationnaire, en utilisant la moyenne de Favre définie par : $\widetilde{\Phi}=\frac{\overline{\rho \Phi}}{\bar{\rho}}$ et en appliquant les hypothèses de la couche limite est le suivant :

Équation de continuité

$$
\frac{\partial}{\partial x}(\bar{\rho} \widetilde{U})+\frac{1}{r} \frac{\partial}{\partial r}(r \bar{\rho} \widetilde{V})=0
$$

Équations de conservation de la quantité de mouvement Équation selon $x$

$\frac{\partial}{\partial x}(\bar{\rho} \widetilde{U} \widetilde{U})+\frac{1}{r} \frac{\partial}{\partial r}(r \bar{\rho} \widetilde{U} \widetilde{V})+\frac{\partial \bar{P}}{\partial x}+\frac{1}{r} \frac{\partial}{\partial r}(r \bar{\rho} \widetilde{u v})-$ Dif.Mol. $=0$

où Dif.Mol est le terme de diffusion moléculaire. Aux grands nombres de Reynolds de la turbulence, les effets moléculaires sont négligeables devant les effets dus à l'agitation turbulente.

Équation selon y

$$
\begin{aligned}
\frac{\partial}{\partial x}(\bar{\rho} \widetilde{U} \widetilde{V})+\frac{1}{r} \frac{\partial}{\partial r}(r \bar{\rho} \widetilde{V} \widetilde{V}) & +\frac{\partial \bar{P}}{\partial r}+\frac{1}{r} \frac{\partial}{\partial r}(r \bar{\rho} \widetilde{v v}) \\
& -\bar{\rho} \frac{\widetilde{w w}}{\widetilde{r}}-\text { Dif.Mol. }=0
\end{aligned}
$$

Équation de conservation du scalaire

$$
\frac{\partial}{\partial x}(\bar{\rho} \widetilde{U} \widetilde{F})+\frac{1}{r} \frac{\partial}{\partial r}(r \bar{\rho} \widetilde{V} \widetilde{F})-\frac{1}{r} \frac{\partial}{\partial r}(r \bar{\rho} \widetilde{v f})+\text { Dif.Mol. }=0
$$

Liaison macroscopique moyenne densité-scalaire :

La relation liant le scalaire responsable des variations de densité à celle-ci s'exprime sous la forme suivante :

$$
\frac{1}{\bar{\rho}}=\frac{\widetilde{F}}{\rho_{\mathrm{j}}}+\frac{1-\widetilde{F}}{\rho_{\mathrm{a}}}
$$

Dans le cas d'un mélange binaire de gaz, $\widetilde{F}$ représente la fraction massique moyenne. Dans le cas d'un mélange d'un gaz chaud dans le même gaz froid, $\widetilde{F}$ représente la température adimensionnée définie par la relation suivante :

$$
\hat{T}=\frac{\widetilde{T}-T_{\mathrm{a}}}{T_{\mathrm{j}}-T_{\mathrm{a}}}
$$

\subsection{Modélisation au second ordre}

Modélisation des équations des tensions de Reynolds :

$$
\frac{\partial}{\partial x} k\left(\bar{\rho} \widetilde{U}_{k} \widetilde{u_{i} u_{\mathrm{j}}}\right)=\widetilde{P}_{i j}+\widetilde{D}_{i j}+\widetilde{\varphi}_{i j}-\frac{2}{3} \delta_{i j} \bar{\rho} \widetilde{\varepsilon}
$$

Termes de production

$$
\widetilde{P}_{i j}=-\bar{\rho}\left(\widetilde{u_{i} u_{k}} \frac{\partial \widetilde{U}_{j}}{\partial x_{k}}+\widetilde{u_{j} u_{k}} \frac{\partial \widetilde{U}_{i}}{\partial x_{k}}\right)
$$

Ce sont des termes exacts qui ne nécessitent aucune modélisation. Ils jouent un rôle important dans la représentation des divers mécanismes de création des contraintes turbulentes.

Terme de diffusion

Ce terme est modélisé comme suit, Sanders [5] :

$$
\widetilde{D}_{i j}=C_{S} \frac{\partial}{\partial x_{k}}\left[\left(\bar{\rho} \frac{\widetilde{k}}{\widetilde{\varepsilon}} \widetilde{u_{k} u_{l}}+\bar{\rho} v \delta_{k l} \frac{\partial}{\partial x_{l}}\left(\widetilde{u_{i} u_{j}}\right)\right]\right.
$$

Terme de corrélation pression-déformation

La modélisation de ce terme est basée sur l'équation de Poisson reliant la pression fluctuante au champ de vitesse.

$$
\widetilde{\varphi}_{i j}=\overline{p^{\prime \prime}\left(\frac{\partial u_{i}^{\prime \prime}}{\partial x_{j}}+\frac{\partial u_{j}^{\prime \prime}}{\partial x_{i}}\right)}=\widetilde{\varphi}_{i j}^{(1)}+\widetilde{\varphi}_{i j}^{(2)}
$$

Le premier terme dit terme de Rotta [14], qui représente la tendance de retour à l'isotropie, a été modélisé en premier lieu par celui-ci comme :

$$
\widetilde{\varphi}_{i j}^{(1)}=-C_{1} \bar{\rho} \widetilde{\varepsilon}\left(\frac{\widetilde{u_{i} u_{j}}}{\widetilde{k}}-\frac{2}{3} \delta_{i j}\right)=-C_{1} \bar{\rho} \widetilde{\varepsilon} a_{i j}
$$

Le deuxième terme concernant l'interaction entre le mouvement moyen et la turbulence, dit aussi terme rapide, est modélisé en utilisant le modèle plus simple, Sanders [5] et Debaty [15], dit modèle $I P$ (isotropisation de la production), défini par :

$$
\widetilde{\varphi}_{i j}^{(2)}=-C_{2}\left(\widetilde{P}_{i j}-\frac{2}{3} \delta_{i j} \widetilde{P}_{k}\right)
$$

où, $\widetilde{P}_{k}=\frac{1}{2} \widetilde{P}_{k k}=-\bar{\rho} \widetilde{u_{i} u_{k}} \frac{\partial \widetilde{U}_{i}}{\partial x_{k}}$ est le taux moyen de production de l'énergie cinétique de la turbulence.

L'ajustement du couple de constantes $\left(C_{1}, C_{2}\right)$ est basé sur certaines expériences faites par certains chercheurs, Debaty [15].

Modélisation de l'équation de dissipation dynamique

L'équation modélisée est de la forme suivante :

$$
\frac{\partial}{\partial x_{k}}\left(\bar{\rho} \widetilde{U_{k}} \widetilde{\varepsilon}\right)=\widetilde{D}_{\varepsilon}+\bar{\rho} \frac{\widetilde{\varepsilon}^{2}}{\widetilde{k}} \widetilde{\psi}(\varepsilon)
$$

Le terme $\widetilde{\psi}(\varepsilon)$ contient tous les effets de production et de destruction de la dissipation dynamique. Ce terme 
Tableau 1. Termes source pour le modèle du second ordre dans l'équation (17).

\begin{tabular}{|c|c|}
\hline Variable & $S_{\Phi}$ \\
\hline$\widetilde{u u}$ & $-2\left(1-C_{2}\right) \bar{\rho} \widetilde{u v} \frac{\partial \widetilde{U}}{\partial r}-\frac{2}{3} \bar{\rho} \widetilde{\varepsilon}-C_{1} \bar{\rho} \widetilde{\tilde{\varepsilon}}\left(\widetilde{u u}-\frac{2}{3} \widetilde{k}\right)+\frac{2}{3} C_{2} \widetilde{P}$ \\
\hline$\widetilde{v v}$ & $-\frac{2}{3} \bar{\rho} \widetilde{\varepsilon}-C_{1} \bar{\rho} \frac{\widetilde{\varepsilon}}{k}\left(\widetilde{v v}-\frac{2}{3} \widetilde{k}\right)+\frac{2}{3} C_{2} \widetilde{P}-\frac{2 C_{s}}{r^{2}} \frac{\widetilde{k}}{\widetilde{\varepsilon}} \widetilde{\rho w w}(\widetilde{v v}-\widetilde{w w})$ \\
\hline$\widetilde{w w}$ & $-\frac{2}{3} \bar{\rho} \widetilde{\varepsilon}-C_{1} \bar{\rho} \frac{\widetilde{\varepsilon}}{k}\left(\widetilde{w w}-\frac{2}{3} \widetilde{k}\right)+\frac{2}{3} C_{2} \widetilde{P}+\frac{2 C_{s}}{r} \frac{\widetilde{k}}{\tilde{\varepsilon}} \bar{\rho} \widetilde{w w}(\widetilde{v v}-\widetilde{w w})$ \\
\hline$\widetilde{u v}$ & $-\left(1-C_{2}\right) \tilde{\rho} \widetilde{v v} \frac{\partial \widetilde{U}}{\partial r}-C_{1} \bar{\rho} \frac{\widetilde{\varepsilon}}{k} \widetilde{u v}-\frac{C_{s}}{r^{2}} \frac{\widetilde{k}}{\tilde{\varepsilon}} \widetilde{\rho} \widetilde{w w} \widetilde{u v}$ \\
\hline$\widetilde{\varepsilon}$ & $\frac{\widetilde{\varepsilon}}{k}\left(C_{\varepsilon, 1} \widetilde{P}-C_{\varepsilon, 2} \bar{\rho} \widetilde{\varepsilon}\right)$ \\
\hline
\end{tabular}

Le coefficient de diffusion turbulente $D$ est $C_{\text {diff }} \frac{\widetilde{k}}{\tilde{\varepsilon}} \widetilde{v v}$. Pour toutes les tensions de Reynolds $C_{\text {diff }}=C_{s}$ et pour le taux de dissipation dynamique $C_{\text {diff }}=C_{\varepsilon}$.

Tableau 2. Constantes du modèle de turbulence de second ordre (RSM).

\begin{tabular}{cccccc}
\hline$C_{1}$ & $C_{2}$ & $C_{s}$ & $C_{\varepsilon, 1}$ & $C_{\varepsilon, 2}$ & $C_{\varepsilon}$ \\
\hline 2,3 & 0,6 & 0,22 & 1,45 & 1,90 & 0,18 \\
\hline
\end{tabular}

est modélisé en utilisant la forme proposée par Launder et al. [16] :

$$
\widetilde{\psi}(\varepsilon)=C_{\varepsilon 1} \frac{\widetilde{P}_{k}}{\bar{\rho} \widetilde{\varepsilon}}-C_{\varepsilon 2}
$$

La modélisation du terme de diffusion $\widetilde{D}_{\varepsilon}$ est donnée par la relation suivante :

$$
\widetilde{D_{\varepsilon}}=C_{\varepsilon} \frac{\partial}{\partial x_{k}}\left[\bar{\rho} \frac{\widetilde{k}}{\widetilde{\varepsilon}} \widetilde{u_{k} u_{l}} \frac{\partial}{\partial x_{l}}(\widetilde{\varepsilon})\right]
$$

\section{Modélisation du terme du flux turbulent du scalaire}

Ce terme est approximé avec l'hypothèse de transport par le gradient :

$$
\widetilde{u_{i} f}=-\frac{v_{t}}{P r_{t}} \frac{\partial \widetilde{F}}{\partial x_{i}}
$$

avec $v_{t}=C_{\mu} \frac{k^{2}}{\varepsilon}$ et $C_{\mu}=0,09$

\subsection{Expression de l'équation générale et des termes sources}

Les équations de transport citées précédemment et dans un système de coordonnées cylindro-polaire peuvent se mettre sous la forme générale parabolique suivante :

$$
\frac{\partial}{\partial x}(\bar{\rho} U \widetilde{\Phi})+\frac{1}{r} \frac{\partial}{\partial r}(r \bar{\rho} V \widetilde{\Phi})=\frac{1}{r} \frac{\partial}{\partial r}\left(r \bar{\rho} D \frac{\partial \widetilde{\Phi}}{\partial r}\right)+S_{\Phi}
$$

Les termes sources $S_{\Phi}$ sont donnés dans le tableau 1 , où $\widetilde{P}=-\bar{\rho} \widetilde{u v} \frac{\partial \widetilde{U}}{\partial r}$.

Les constantes utilisées dans la présente étude sont données dans le tableau 2.

\section{Méthode numérique}

Les équations à résoudre, de Navier-Stokes, de conservation de l'énergie, auxquelles sont ajoutées celles correspondant au modèle de turbulence modélisées précédemment sont toutes des équations du type convection diffusion avec termes sources. Chaque équation de la variable $\Phi$ pour un écoulement stationnaire bidimensionnel peut être donc mise sous la forme de l'équation (17). Cette équation est intégrée avec les conditions aux limites appropriées selon la méthode de volumes finis décrite par Patankar [17] sur un volume de contrôle centré sur le lieu où est définie la variable $\Phi$. Pour obtenir la meilleure résolution possible, les équations sont discrétisées sur un maillage décalé où les vitesses et les flux turbulents scalaires sont localisés sur les faces du volume de contrôle, le cisaillement turbulent sur les sommets tandis que les variables scalaires sont sur les points centraux (nœuds).

Du fait du couplage et de la non-linéarité des équations à résoudre, la stabilité du processus itératif ne peut être assurée que par l'utilisation de coefficients de sous-relaxation et la linéarisation des termes sources. Un maillage décalé et suffisamment fin permet aussi l'augmentation de la stabilité numérique. La convergence est atteinte lorsque la somme des résidus (défaut de bilan de chaque équation résolue) dans tout le domaine devient inférieure à une valeur test suffisamment petite.

Dans le but de diminuer ce temps de calcul, plusieurs schémas numériques sont possibles comme, entre autres, les schémas upwind, hybride et puissance, Patankar [17] et Luppes [18]. Parmi ces schémas, le schéma puissance (PLDS, pour power law difference scheme) n'est pas celui qui donne le meilleur gain de temps mais c'est celui qui offre la solution la plus proche de la solution exacte donnée par le schéma exact (LEDS, pour locally exact difference scheme), Ruffin [6]. C'est celui que nous avons utilisé dans nos calculs.

\subsection{Justification du choix du schéma d'interpolation PLDS}

Pour évaluer la variable $\Phi$, plusieurs schémas numériques, cités au paragraphe précédent, sont possibles. Bien 


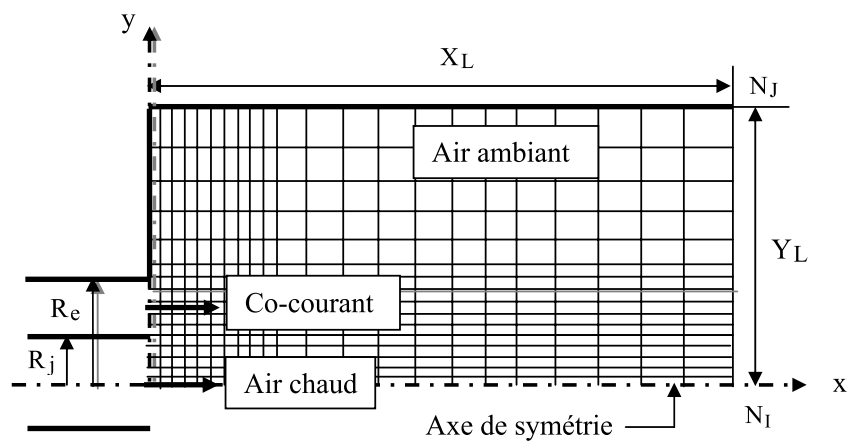

Fig. 1. Configuration des jets axisymétriques.

que le schéma exponentiel donne la solution exacte pour n'importe quelle valeur du nombre de Peclet, il n'est en fait pas utilisé tel quel, car d'une part, les exponentielles sont coûteuses à calculer et d'autre part il n'est pas exact pour les problèmes bidimensionnels ou tridimensionnels. Ce sont plutôt les schémas hybride et puissance, des versions approchées du schéma exponentiel, qui sont employés.

Dans le schéma hybride, les coefficients des équations discrétisées font l'objet d'une approximation linéaire par morceaux suivant la valeur du nombre de Peclet. Dans le schéma puissance, ces coefficients sont approchés par une fonction polynomiale du nombre de Peclet. Le schéma puissance est donc plus précis que le schéma hybride, voir la référence (Patankar [17] pour plus de détails).

\subsection{Configuration étudiée}

Dans cette présente étude, nous allons nous intéresser aux jets axisymétriques turbulents confinés non réactifs. Nous avons adapté notre code de calcul sur une configuration moins complexe et la plus répandue en études soit théoriques ou expérimentales, cette configuration est celle représentée sur la figure 1.

La valeur du nombre de Reynolds à l'éjection $R e_{\mathrm{j}}$ est maintenue suffisamment élevée pour que l'écoulement soit pleinement turbulent.

\subsection{Maillage adopté et domaine de calcul}

Le maillage, comprenant 10000 nœuds (100 nœuds axialement et 100 nœuds radialement), est de type variable, qui présente deux zones de resserrement de mailles de calcul : la première, radialement, jusqu'à 20 fois le rayon du jet principal, et la deuxième axialement dans la zone d'éjection $\left(x / R_{\mathrm{j}}<20\right)$. Pour étudier la sensibilité de la solution vis-à-vis du maillage, on a mené plusieurs essais en augmentant successivement la densité du maillage. Les deux figures $2 \mathrm{a}$ et $\mathrm{b}$ montrent respectivement, les évolutions axiales de la vitesse longitudinale et du scalaire pour les cinq maillages testés. La solution ne change pas de manière significative. On peut donc conclure qu'elle est indépendante du maillage.

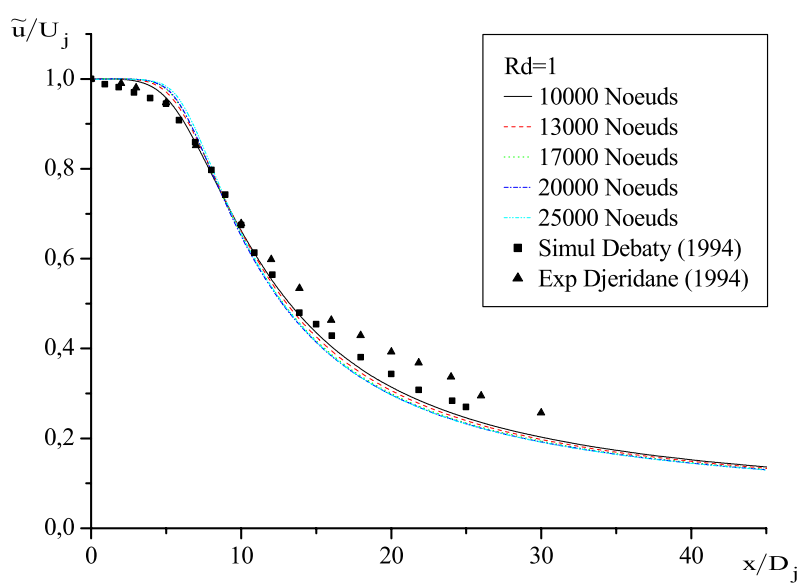

(a)

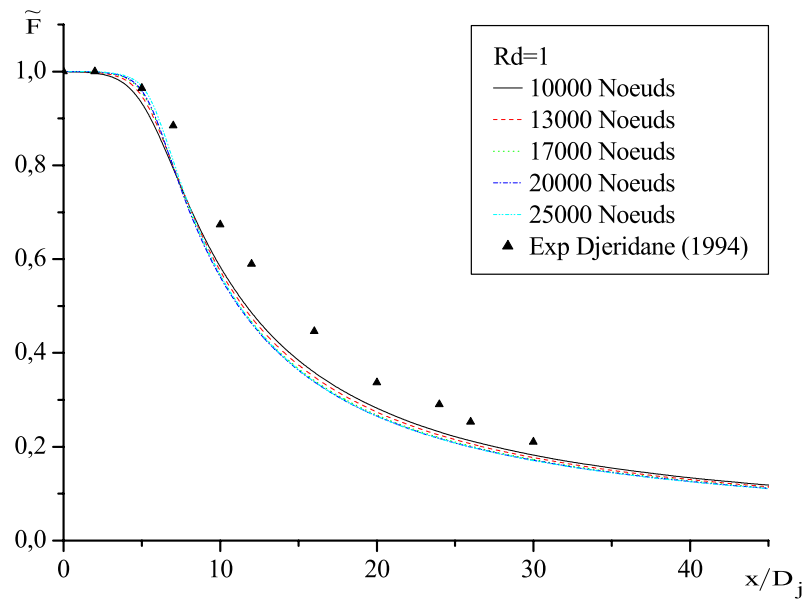

(b)

Fig. 2. (a) Effet du maillage : évolution de la vitesse longitudinale moyenne sur l'axe du jet. (b) Effet du maillage : évolution du scalaire sur l'axe du jet.

Le domaine de calcul a une longueur $X_{L}=1 \mathrm{~m}$ ce qui équivaut à 125 fois le diamètre du jet principal $\left(R_{\mathrm{j}}\right)$ et 7 fois le diamètre du jet secondaire $(R e)$, et une hauteur $Y_{L}=0,16 \mathrm{~m}$ ce qui équivaut à 20 fois le diamètre du jet principal.

\section{Résultats numériques et discussion}

Nous allons à présent, exposer et discuter les résultats obtenus avec notre modèle en comparaison avec ceux issus des expériences de Djeridane [1] et de la simulation numérique de Sanders [5].

Afin d'interpréter plus facilement les résultats, toutes les variables sont adimensionnées par les grandeurs imposées à la section d'éjection du jet $\left(U_{\mathrm{j}}, T_{\mathrm{j}}, D_{\mathrm{j}}\right)$.

\subsection{Conditions aux limites}

En amont, les profils d'entrée sont donnés par l'expérience de Chassaing [19]. Le profil de la dissipation de 
Tableau 3. Paramètres à l'émission des écoulements jets.

\begin{tabular}{cccccc}
\hline$T_{\mathrm{j}}(\mathrm{K})$ & 200 & 305 & 600 & 1500 & 2143 \\
\hline$R_{d}$ & 1,5 & 0,98 & 0,5 & 0,20 & 0,14 \\
\hline$U_{\mathrm{j}}\left(\mathrm{m} . \mathrm{s}^{-1}\right)$ & 32 & 38 & 54 & 85 & 103 \\
\hline$M_{\mathrm{j}}(\mathrm{N})$ & 0,09 & 0,09 & 0,09 & 0,09 & 0,09 \\
\hline
\end{tabular}

l'énergie cinétique de la turbulence est donné par une relation basée sur l'hypothèse d'une couche limite turbulente en équilibre, Pope [20] : $\varepsilon=0,09^{(3 / 4)} \times \frac{k^{3 / 2}}{0,43\left(0,5 D_{j}-r\right)} \times C$. Pour prendre en considération la variation de la masse volumique dans les jets turbulents à masse volumique variable, on a introduit le coefficient : $C=0,66+0,26 \ln R_{d}$, Ruffin [6]. Sur l'axe de symétrie, la plupart des variables admet perpendiculairement à l'axe une dérivée nulle. À la sortie, on impose un gradient nul pour chaque variable. La prise en compte de paroi est obtenue par l'utilisation des fonctions de paroi. Cette approche, très utilisée dans les écoulements à grand nombre de Reynolds, est économique, robuste, et raisonnablement précise.

Enfin la valeur du scalaire $F$ est égale à 1 dans le tube et 0 ailleurs.

\subsection{Paramètres gardés constants lors des simulations}

Il existe différentes stratégies lorsque l'on mène des études sur des jets turbulents à masse volumique variable. On peut maintenir constants différents paramètres comme le débit, le flux de quantité de mouvement, le nombre de Reynolds, le rapport des vitesses. Il semble, d'après Djeridane [1] et Ruffin [6], que le flux de quantité de mouvement est apparemment le bon pour étudier les effets de variations de la masse volumique. Ainsi, et pour cerner exclusivement les effets de variations de la masse volumique, c'est donc la vitesse d'émission du jet principal (air chauffé) qui varie, afin de maintenir constant, d'un calcul à l'autre, le flux de quantité de mouvement. La vitesse du jet secondaire (co-courant) est maintenue constante et prise égale à : $U c c=1 \mathrm{~m} \cdot \mathrm{s}^{-1}$. En outre, nous avons pris une valeur nulle pour l'accélération de la pesanteur. Les valeurs du nombre de Froude $(F r)$ sont maintenues suffisamment élevées pour pouvoir négliger les termes de gravité. D'après Sanders [5] et pour un nombre de Froude $F r=14000\left(R_{d}=0,14\right)$, l'effet de flottabilité ne se manifeste qu'à partir de $X / D \mathrm{j} \geq 50$. Les valeurs du nombre de Froude pour $R_{d}=0,14$ et $R_{d}=1$ sont ici égales respectivement à $F r_{0,14}=22006$ et $F r_{1}=901580$. Les divers paramètres des écoulements calculés sont rassemblés dans le tableau 3.

\subsection{Champ de vitesse}

La figure $3 a$ montre les profils axiaux de la vitesse longitudinale moyenne du jet. On peut voir que le développement axial de la vitesse longitudinale moyenne est bien influencé par le rapport de densité $R_{d}$. Ce

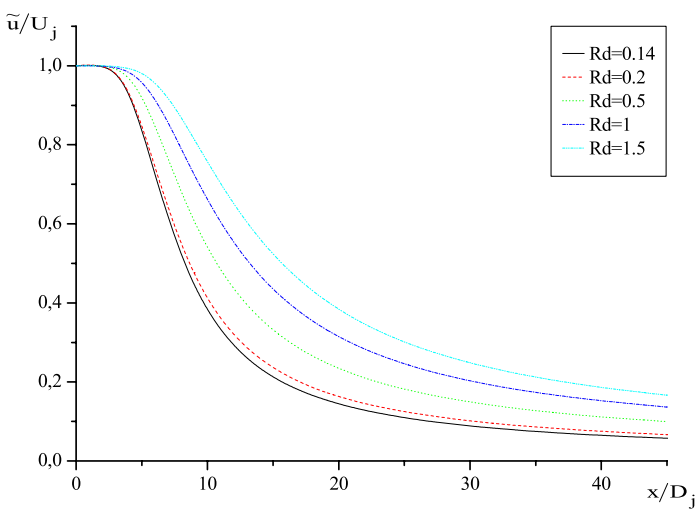

(a)

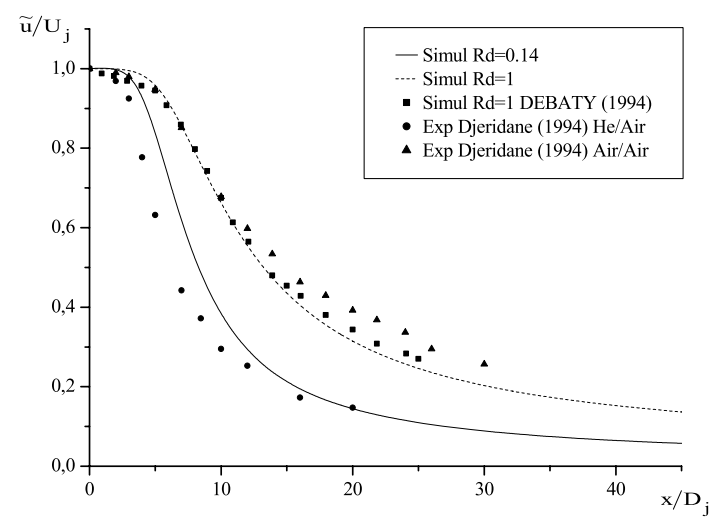

(b)

Fig. 3. (a) Effets de densité sur l'évolution de la vitesse longitudinale moyenne sur l'axe du jet. (b) Effets de densité sur l'évolution de la vitesse longitudinale moyenne sur l'axe du jet. Comparaison : simulation-expérience, $R_{d}=1$ et $R_{d}=0,14$.

développement axial montre une décroissance plus prononcée de la vitesse longitudinale moyenne $\widetilde{U}_{c}$ lorsque le rapport de densité $R_{d}$ diminue, plus $R_{d}$ est faible, plus le jet se développe beaucoup plus vite. Cela est dû à la conservation de la quantité de mouvement : quand $R_{d}<1$, la masse volumique augmente en fonction de la distance axiale et par conséquent la vitesse diminue plus rapidement qu'avec $R_{d}=1$. L'évolution de la vitesse moyenne montre que le profil est composé de trois régions différentes :

- La première, très proche de la buse d'éjection où la variation de la vitesse est quasiment insensible et où prédominent les forces d'inertie. Cette région, dite « région du cône potentiel » est de plus en plus courte lorsque le rapport de densité $R_{d}$ diminue, elle se rapproche donc de la buse d'éjection pour les gaz légers.

- Une deuxième région, dont l'évolution, comme pour un jet incompressible, est quasiment hyperbolique et où la vitesse commence à diminuer à partir la première région.

- Enfin, une troisième région où la vitesse est asymptotique, et par conséquent la variation de la vitesse reste très faible. 
Cependant, la masse volumique ne serait pas la seule cause d'un tel effet. Gladnick et al. [21] montrent, pour des jets coaxiaux, que la décroissance axiale de la vitesse longitudinale est liée au rapport initial $\left(U_{\mathrm{j}} / U_{\mathrm{e}}\right)$ ainsi qu'à la forme du profil de la vitesse initiale. Le nombre de Reynolds et le confinement sont aussi des paramètres à prendre en considération, Djeridane [1]. D'après ce dernier, l'existence d'un co-courant et/ou d'un confinement retarde légèrement l'épanouissement d'un jet.

Sur la figure $3 \mathrm{~b}$, on remarque et dans le cas de jet d'air, $R_{d}=1$, une sous-estimation des résultats expérimentaux de Djeridane [1] par la simulation numérique dans la région relativement loin de la buse d'éjection, à partir de $X=10 \times D \mathrm{j}$. Comparés à la simulation numérique de Debaty [15], les résultats sont en très bon accord. Cette sous-estimation des résultats n'est cependant pas importante et les résultats numériques sont assez proches de ceux de l'expérience. Pour les gaz légers, $R_{d}=$ 0,14 , la simulation surestime légèrement les résultats de l'expérience dans la région proche de la buse d'éjection, $3 \leq X / D \mathrm{j} \leq 10$, figure $3 \mathrm{~b}$. Cet écart entre la simulation et les résultats expérimentaux peut être dû aux conditions en amont qui influencent beaucoup la zone initiale du jet et/ou à la modélisation utilisée dans cette présente étude.

\section{4 Écart-type des fluctuations de vitesse}

Les figures 4a, 5a représentent l'évolution axiale de l'écart-type des fluctuations de vitesse longitudinale et radiale. Ces deux quantités suivent des évolutions quasisimilaires en fonction du rapport de densité $R_{d}$ bien que les intensités de $v^{\prime}$ sont moins fortes que celles de $u^{\prime}$. L'influence de la masse volumique est, là, encore importante. Cette évolution permet de dégager deux zones distinctes associées à la croissance et la décroissance respectivement de $u^{\prime}$ et de $v^{\prime}$, qui sont d'autant plus prononcées que le rapport de densité $R_{d}$ est faible. En outre, les valeurs maximales atteintes sont en amplitude et en position axiale différentes, autrement dit : plus $R_{d}$ est faible, plus l'intensité de la turbulence est plus forte et plus rapide. Cette intensité se trouve légèrement augmentée en présence d'un confinement, Djeridane [1]. Les décroissances observées (Figs. 4a, 5a) semblent être de type hyperbolique et donc analogue aux décroissances de la vitesse longitudinale moyenne.

Comparé aux résultats expérimentaux de Djeridane [1], on remarque sur la figure $4 \mathrm{~b}$, pour $R_{d}=1$, un écart relativement important, en amplitude, des valeurs maximales de $u^{\prime}$. Ces valeurs sont atteintes à la même position axiale que celles de l'expérience. Dans le cas des gaz légers $R_{d}=0,14$, la figure $4 \mathrm{c}$, les valeurs maximales atteintes sont en amplitude et positions axiales différentes. Les mêmes observations peuvent être faites sur l'évolution de $v^{\prime}$ (Figs. 5b, c). Ceci pourrait être dû à différents effets présents dans la section d'éjection ou à l'intérieur même du tube. L'unicité des profils à l'émission, par exemple, le nombre de Reynolds, peuvent être parmi les facteurs responsables de la différence entre l'expérience et la simulation numérique

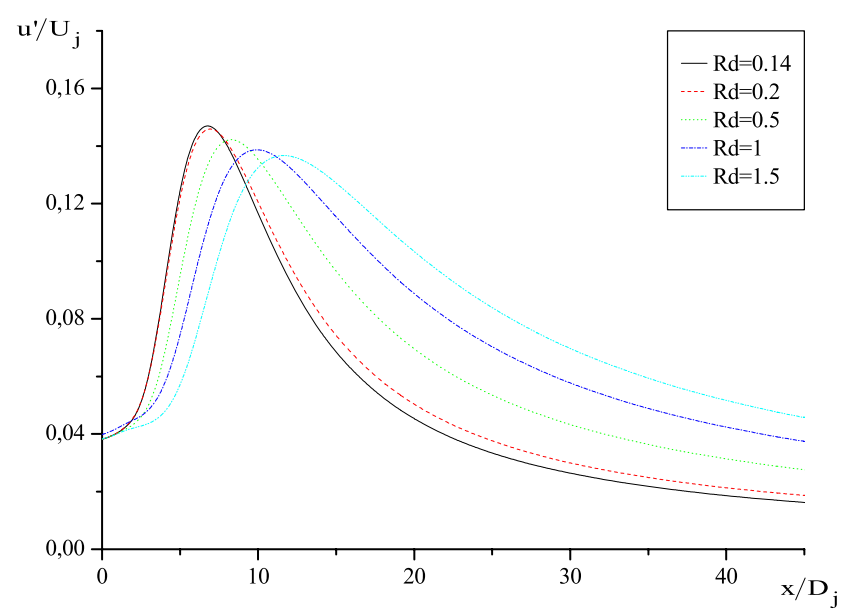

(a)

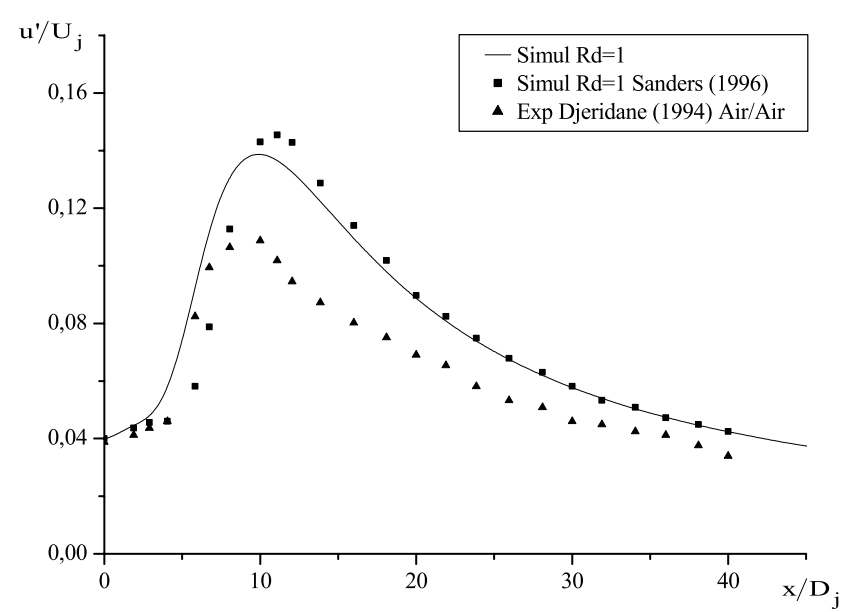

(b)

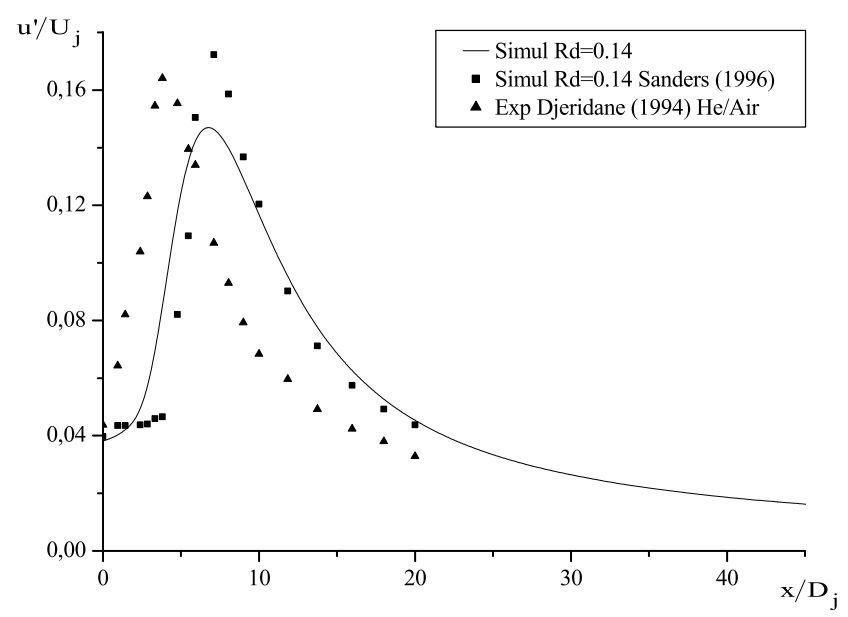

(c)

Fig. 4. (a) Effets de densité sur l'évolution de l'écart-type de la vitesse longitudinale sur l'axe du jet. (b) Effets de densité sur l'évolution de l'écart-type de la vitesse longitudinale sur l'axe du jet. Comparaison : simulation-expérience, $R_{d}=1$. (c) Effets de densité sur l'évolution de l'écart-type de la vitesse longitudinale sur l'axe du jet. Comparaison : simulation-expérience, $R_{d}=0,14$. 


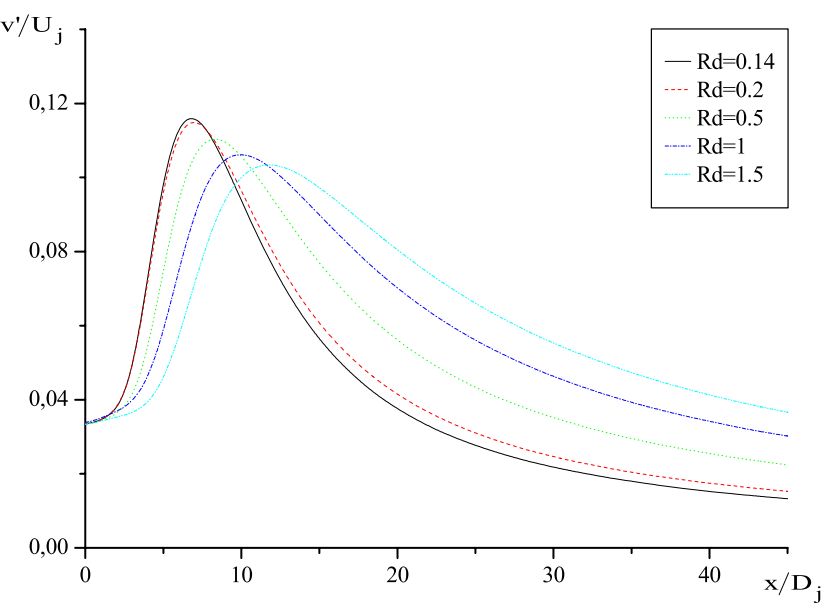

(a)

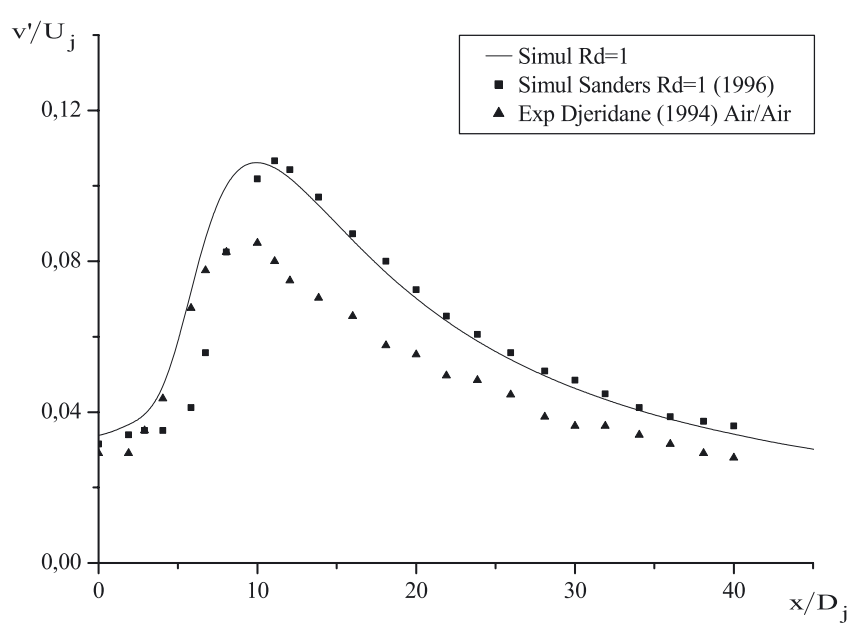

(b)

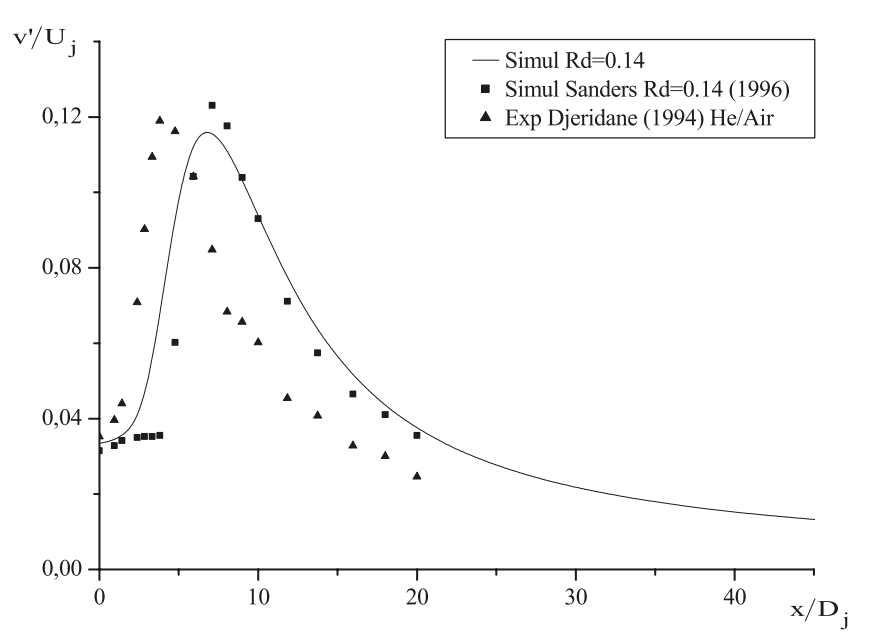

(c)

Fig. 5. (a) Effets de densité sur l'évolution de l'écart-type de la vitesse radiale sur l'axe du jet. (b) Effets de densité sur l'évolution de l'écart-type de la vitesse radiale sur l'axe du jet. Comparaison : simulation-expérience, $R_{d}=1$. (c) Effets de densité sur l'évolution de l'écart-type de la vitesse radiale sur l'axe du jet. Comparaison : simulation-expérience, $R_{d}=0,14$.

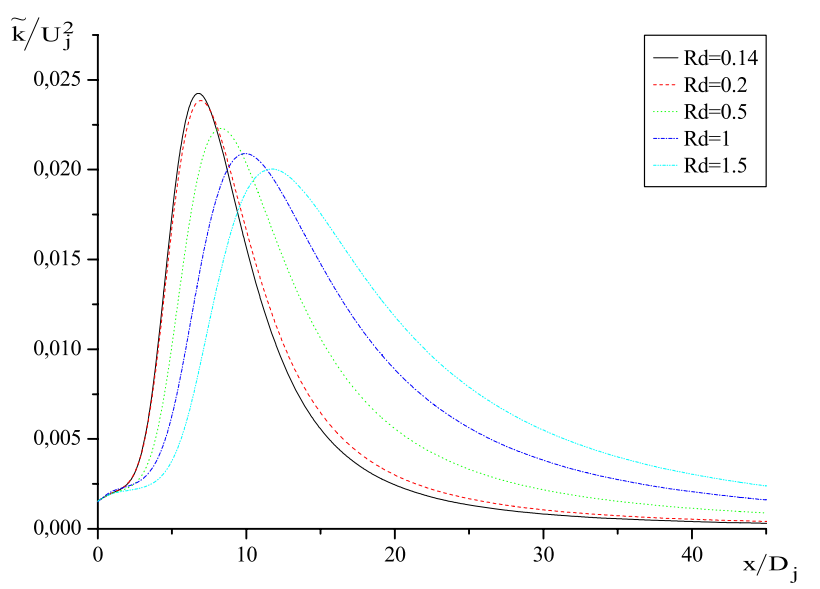

(a)

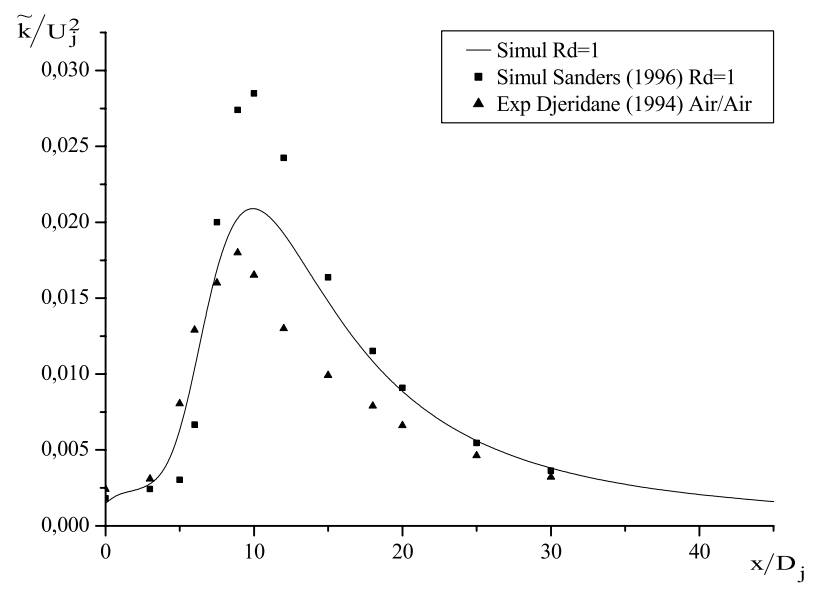

(b)

Fig. 6. (a) Effets de densité sur l'évolution de l'énergie cinétique de la turbulence sur l'axe du jet. (b) Effets de densité sur l'évolution de l'énergie cinétique de la turbulence sur l'axe du jet. Comparaison : simulation-expérience, $R_{d}=1$.

plus particulièrement dans le cas de $R_{d}=0,14$. La modélisation utilisée est aussi un autre facteur à prendre en considération.

Les résultats obtenus par notre simulation prédisent, en général, mieux les résultats expérimentaux que ceux de Sanders [5] plus particulièrement dans la région proche de la buse d'éjection, $X / D_{\mathrm{j}} \leq 10$.

\section{5 Énergie cinétique de la turbulence}

La figure 6a montre l'évolution de l'énergie cinétique de la turbulence moyenne sur l'axe du jet en fonction de la position axiale pour les différents rapports de densité $R_{d}$ d'air chauffé. Cette évolution traduit les mêmes effets observés sur l'évolution axiale de l'écart-type des fluctuations de vitesse longitudinale et radiale. L'influence de $R_{d}$ se manifeste à la fois sur la position du maximum de l'énergie cinétique de la turbulence et sur sa tendance quasi-hyperbolique. En effet, les pics des profils se rapprochent de la buse d'éjection lorsque le rapport 


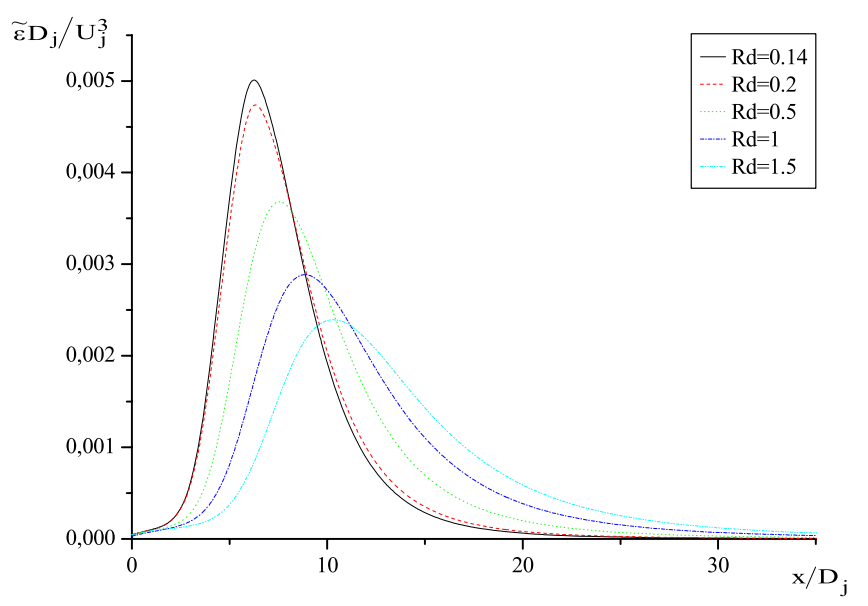

Fig. 7. Effets de densité sur l'évolution du taux de dissipation de l'énergie cinétique de la turbulence sur l'axe du jet.

de densité $R_{d}$ diminue (lorsque le gaz est plus léger) et inversement, étant donné que le mélange est plus rapide. Les pics se situent approximativement à la position à partir de laquelle la vitesse commence à chuter de façon remarquable, ce qui s'explique par le fait que la région du cône potentiel devient de plus en plus courte avec la diminution de $R_{d}$ (jets légers), à cause de leur «bon mélange » avec l'air ambiant. Ceci fait augmenter la densité moyenne conformément au principe de la conservation de quantité de mouvement.

La comparaison avec les résultats expérimentaux, figure $6 \mathrm{~b}$, montre une meilleure prédiction de la simulation numérique comparée à celle de Sanders [5]. Un écart des valeurs maximales en amplitude et en position axiale est très négligeable vis-à-vis de celui obtenu par Sanders.

\subsection{Taux de dissipation de l'énergie cinétique de la turbulence}

L'évolution du taux de dissipation de l'énergie cinétique de la turbulence, figure 7 , est la même que celle observée pour l'énergie cinétique de la turbulence. Le taux de dissipation de l'énergie cinétique de la turbulence croît et décroît rapidement et les pics correspondant aux maximums du taux de dissipation sont décalés vers l'aval par rapport à ceux de l'énergie cinétique, ce qui s'explique par le fait que la région de la buse d'éjection est dominée par les structures de grandes échelles et par conséquent il y a génération de l'énergie cinétique de la turbulence et la dissipation est très faible, et juste après cette région, la dissipation, qui s'effectue à un niveau moléculaire, commence à augmenter transformant ainsi l'énergie cinétique de la turbulence en chaleur.

\subsection{Champ du scalaire}

L'évolution du scalaire représentée sur la figure 8a, montre un comportement analogue à celui de la vitesse longitudinale moyenne.

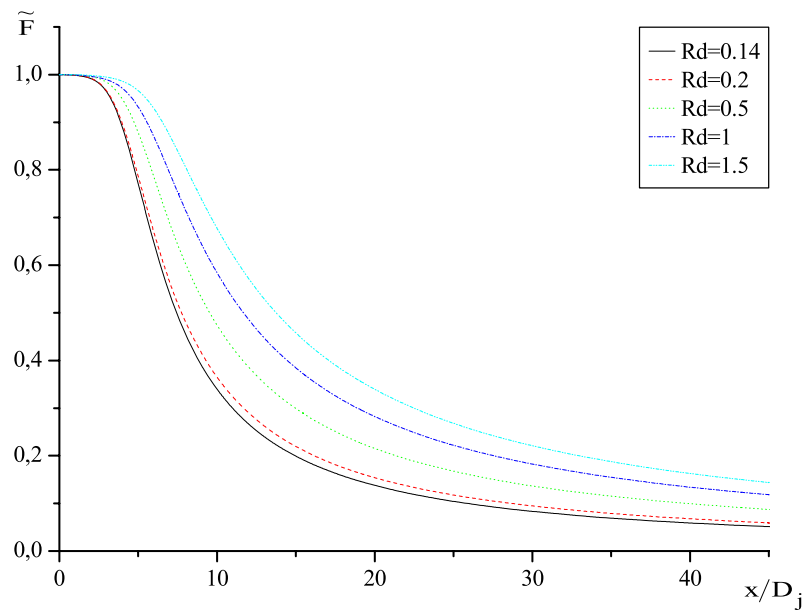

(a)

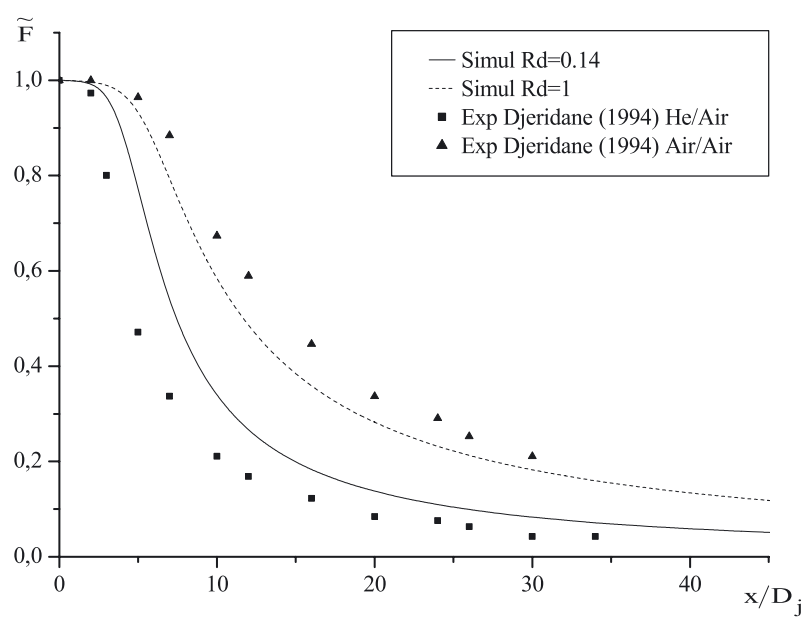

(b)

Fig. 8. (a) Effets de densité sur l'évolution du scalaire sur l'axe du jet. (b) Effets de densité sur l'évolution du scalaire sur l'axe du jet. Comparaison : simulation-expérience, $R_{d}=1$ et $R_{d}=0,14$.

Les résultats expérimentaux de Djeridane [1], figure $8 \mathrm{~b}$, sont surestimés par la simulation numérique. Cette surestimation des résultats n'est cependant pas importante, en particulier dans le cas $R_{d}=1$, et les résultats numériques sont assez proches de ceux de l'expérience.

\section{Conclusion}

Le travail développé dans ce présent article est une approche à l'étude des jets turbulents axisymétriques confinés non réactifs à masse volumique variable avec le modèle de transport à l'ordre deux en moyenne de Favre. L'objectif principal était d'étudier l'influence de la variation de la masse volumique sur le développement du champ dynamique et scalaire de ce type d'écoulements dans la région initiale du jet $\left(X / D_{\mathrm{j}} \leq 40\right)$.

L'étude est réalisée pour des jets turbulents dès l'émission en maintenant constant le flux de quantité de 
mouvement pour les différents rapports de densité. Ainsi, sur la base des résultats obtenus, nous pouvons tirer les conclusions suivantes :

- Le développement initial du champ dynamique d'un jet turbulent à masse volumique est essentiellement gouverné par le rapport de densité $R_{d}$. Les influences de paramètres tels que le nombre de Reynolds, le cocourant et le confinement, ne sont pas prépondérantes. Dans certaines configurations (co-courant et/ou confinement important), ces paramètres peuvent avoir un effet considérable sur la structure de l'écoulement considéré, Djeridane [1] et Pagé [7].

- L'influence de la variation de la densité sur l'entraînement du fluide extérieur par le jet est clairement établie dans la région initiale, puisqu'un gaz plus léger que l'ambiant dans lequel il s'épanouit se mélange plus rapidement, et donc possède un entraînement plus important, qu'un gaz lourd.

- Dans la région proche de la buse d'éjection, $X / D_{\mathrm{j}} \leq 10$, on peut clairement remarquer une amélioration des résultats obtenus qui sont assez proches de l'expérience, plus particulièrement dans le cas $R_{d}=1$. Les profils à l'émission utilisés proviennent de l'expérience, réalisée par Chassaing [19], en jet de tube d'air dans de l'air au repos. Nous pensons que cette amélioration des résultats est due aux profils en amont utilisés dans cette présente étude, en particulier le profil du taux de la dissipation de l'énergie cinétique de la turbulence qui est fonction de la distance radiale.

- Enfin, on peut dire que l'écart qui existe entre les résultats obtenus par le modèle RSM et ceux obtenus par l'expérience de Djeridane [1] pourrait être dû à différents facteurs tels que le choix des conditions à l'amont (non uniques et semi-empiriques), le modèle de turbulence et l'approche numérique adoptés. La région proche de la buse d'éjection est très influencée par les conditions en amont. Les résultats, obtenus avec des modélisations au second ordre relativement simples, sont cependant en générale satisfaisants.

- Afin de mieux comprendre et modéliser la physique des écoulements à masse volumique variable, des formulations objectives et plus élaborées sont nécessaires pour la modélisation des termes les plus importants (par exemple : $\widetilde{\varphi}_{i j}$ et $\widetilde{\psi}(\varepsilon)$ ), présents dans les équations de transport exactes des tensions de Reynolds et de la dissipation dynamique. Des conditions aux limites bien déterminées peuvent aussi apporter des améliorations aux résultats obtenus.

\section{Références}

[1] T. Djeridane, Contribution à l'étude expérimentale de jets turbulents axisymétriques à densité variable, Thèse de doctorat, Univ. Aix-Marseille II, 1994

[2] A. Favre, L.S.G. Kovasznay, R. Dumas, J. Gaviglio, M. Coantic, La turbulence en mécanique des fluides, éd. Gauthiers-Villars, 1976
[3] G. Harran, Analyse physique, modélisation et simulation numérique des mécanismes de mélange dans les jets simple et coaxiaux turbulents, Thèse INPT nº 841, 1994

[4] L. Joly, Écoulements turbulents cisaillés libres à masse volumique variable, Thèse INPT n ${ }^{\circ}$ 944, 1994

[5] H. Sanders, B. Sarh, I. Gokalp, Variable density effects in axisymmetric isothemal turbulent jets: a comparison between a first-and second-order turbulence model, Int. J. Heat Mass Trans. 40 (1997) 823-842

[6] E. Ruffin, Étude de jets turbulents à densité variable à l'aide de modèles de transport au second ordre, Thèse de doctorat, Université d'Aix-Marseille II, 1994

[7] J. Pagé, B. Sarh, I. Gökalp, Étude expérimentale des jets turbulents à masse volumique variable, $5^{\mathrm{e}}$ congrès francophone de vélocimétrie laser, Rouen, 1996

[8] P. Chassaing, R.A. Antonia, F. Anselmet, L. Joly, S. Sarkar, Variable density fluid turbulence, Kluwer Academic Publishers, 2002

[9] S. Gupta, Test matrix for GAMELAN: Experimental characterisation of vertical helium jets or plumes in confined or unconfined geometries, Technical report CEA/DM2S/SFME/LTMF/RT/06-005/A, 2006

[10] H. Sanders, B. Sarh, I. Gokalp, Étude numérique des jets turbulents à température élevée, Rev. Gén. Therm. 35 (1996) 232-242

[11] G. Harran, P. Chassaing, L. Joly, M. Chibat, Étude numérique des effets de densité dans un jet turbulent en microgravité, Rev. Gén. Therm. 5 (1996) 151-176

[12] L. Joly, A. Purwanto, G. Harran, Modélisation des jets turbulents subsoniques fortement chauffés, Rev. Gén. Therm., 1997

[13] J. Mi, D.S. Nobes, G.J. Nathan, Influence of jet exit conditions on the passive scalar field of an axisymmetric free jet, J. Fluid Mech., 2001

[14] J. Rotta, Statistische theorie nichthomogener turbulenz II, Z. Phys. 131 (1951) 51-77

[15] P. Debaty, Performances des modèles de turbulence au second ordre appliqués à des configurations axisymétriques simulées par éléments finis, Thèse de doctorat, École Centrale de Lyon, 1994

[16] B.E. Launder, G.J. Reece, W. Rodi, Progress in the development of a Reynolds-stress turbulence closure, J. Fluid Mech. 68 (1975) 537-566

[17] S.V. Patankar, Numerical heat transfert and fluid flow, Series in computational methods in mechanics and thermal sciences, Hemisphere Publishing Corporation \& Mc Graw Hill B.C., 1980

[18] R. Luppes, The numerical simulation of turbulent jets and diffusion flames, Technische Universiteit Eindhoven Netherlands, pp. 51-55, 2000

[19] P. Chassaing, Mélange turbulent de gaz inertes dans un jet de tube libre, Thèse de doctorat Es-Sciences, INPT, Toulouse, 1979

[20] S.B. Pope, Turbulent flows, Cambridge University Press, 2000, p. 379

[21] P.G. Glandnick, A.C. Enotiadis, J.C. Larue, G.S. Samuelsen, Near-field characteristics of a turbulent coflowinng jet, AIAA J. 28 (1990) 1405-1414 